Z. klin. Chem. u. klin. Biochem.

9. Jg., S. 375-382, September 1971

\title{
Untersuchungen über die Rh-(D)-Eigenschaft eines kleinmolekularen Erythrocytenmembranproteins mit der SchulTz-DALE-Reaktion ${ }^{1}$ )
}

\author{
Von H. WeICKer, J. Kropp 2 ) und D. RoelCKe \\ Aus der S. J. Thannbauser-Abteilung für Stoffivechseluntersucbungen (Vorstand: Prof. Dr. H. Weicker) der Medizinischen \\ Universitäts-Poliklinik Heidelberg und dem Institut für Immunologie und Serologie (Direktor: Prof. Dr. E. Krab) der \\ Universität Heidelberg
}

(Eingegangen am 30. März 1971)

\begin{abstract}
Mit einer gut reproduzierbaren Methode konnten wir aus Rh-positiven Erythrocyten cin kleinmolekulares Membranprotein isolieren, über dessen biochemische Eigenschaften in einer ersten Mitteilung ausführlich berichtet wurde. In 30 SCHULTZ-DALE-Versuchen sowohl nach aktiver Immunisierung von Meerschweinchen mit Rh-positiven Erythrocyten als auch nach passiver Immunisierung in vivo und passiver Sensibilisierung in vitto mit gereinigtem Anti-D konnte durch dieses Protein eine Muskelkontraktion am Uterushornsegment des Versuchstieres ausgelöst werden. Diese Reaktion mit nachfolgender Desensibilisierung erhielten wir nur nach Applikation von korrespondierenden Antigenen. Die SchuLtz-DaLE-Reaktion war durch Antihistaminika zu hemmen. Unspezifische Muskelkontraktionen konnten durch Vergleichsuntersuchungen an nichtsensibilisierten Versuchstieren ausgeschlossen werden. Bei Verwendung von Human-Albumin, Rinderalbumin und Proteinen von rh-negativen Erythrocyten traten keine anaphylaktischen Reaktionen ein. In dem Albumin-Antialbumin-System oder nach aktiver Immunisierung mit rh-negativen Erythrocyten konnte durch das Protein von Rh-positiven Erythrocyten keine Muskelkontraktion oder Desensibilisierung ausgelöst werden. Unter standardisierter Versuchsanordnung, bei der $\mathrm{pH}, \mathrm{Tem}-$ peratur und Sauerstoffsättigung im Muskelbad konstant waren und keine spontanen Kontraktionen der Uterusmuskulatur beobachtet wurden, fanden wir gut reproduzierbare Resultate. Der Hämagglutinations-Hemmtest und die passive Hämagglutination sowie die Immunpräzipitation in der OuCHTERLONY-Technik eigneten sich bei dem niedrigen Molekulargewicht und der zur Isolierung notwendigen Gefriertrocknung des Proteins nicht zum Antigen-Nachweis. Die Ergebnisse der ScHuLTz-DALE-Methode jedoch ergaben einen berechtigten Hinweis auf die $\mathrm{D}$-Antigen-Eigenschaften des Membranproteins, isoliert von Rh-positiven Erythrocyten.
\end{abstract}

\section{Studies on the $R b-(D)$-properties of a low molecular weight erytbrocyte membrane protein, using the Schultz-Dale reaction}

A low molecular weight membrane protein has been isolated reproducibly from $\mathrm{Rh}$-positive erythrocytes and its biochemical properties have been reported in detail in a previous communication. The protein caused muscle contraction of the uterus horn segment of the guinea pig in 30 Schultz-DALE tests, and also after the active immunisation of guinea pigs with Rh-positive erythrocytes, and after passive immunisation in vivo and passive sensitisation in vitro with purified anti-D. The latter reaction with subsequent desensitisation was only observed after the application of the corresponding antigens. The ScHuLrz-Dale reaction was inhibited by antihistamines. The possibility of unspecific muscle contraction was excluded by control experiments with non-sensitised animals. Human albumin, bovine albumin and proteins from th-negative erythrocytes did not cause an anaphylactic reaction. In the albumin-antialbumin system, or after active immunisation with rh-negative erythrocytes, the protein from Rh-positive erythrocytes did not cause muscle contraction or desensitisation. The results showed good reproducibility under standardised experimental conditions, with constant $\mathrm{pH}$, temperature and $\mathrm{O}_{2}$ saturation in the muscle incubation bath; there was no observable spontaneous contracion of the uterus muscle. Neither the haemagglutination-inhibition test, the passive haemagglutination, nor the OUCHTERLONY immunoprecipitation are suitable for the antigen detection, owing to the low molecular weight and the freeze drying that is necessary for the isolation of the protein. The SCHULTZ-DALE method, however, gave a valid indication of the $\mathrm{D}$-antigen propertics of the membrane protein isolated from Rh-positive erythrocytes.

\section{Einleitung}

Über die Isolierung und biochemische Charakterisierung eines kleinmolekularen Membranproteins, das wir von Rh-positiven Erythrocyten durch ein Anti-D-Immunadsorptionsverfahren erhalten hatten, wurde in einer ersten Mitteilung berichtet (1). Dieses Protein hatte ein Molekulargewicht zwischen 6000 und 10000 und stellte sich bei Gelfiltration, DEAE-Ionenaustauscherchromatographie, Diskelektrophorese, Ultrazentrifugation und bei Dünnschichtchromatographie als einheitliche Fraktion dar. Es enthielt keine Kohlenhydrate, Cholesterin, Triglyceride oder Lecithin. Der Peptidgehalt war durch die Biuret-Reaktion und das IR-Spektrum

1) Mit Unterstützung der Deutschen Forschungsgemeinschaft.

2) Enthält Teile der Dissertation von J. KROpr. bei einem Stickstoffgehalt von $14,6 \%$ gesichert. Der $\mathrm{Na}^{+}, \mathrm{K}^{+}$- und $\mathrm{Ca}^{++}-$Gehalt lag unter $0,2 \%$. In den qualitativen und quantitativen Aminosäureanalysen des hydrolysierten Proteins fanden wir 14 Aminosäuren. $\mathrm{Da}$ die Antigen-Eigenschaften des Proteins bei seinem niedrigen Molekulargewicht durch Immunpräzipitation, Hämagglutinations-Hemmtest und andere serologische Methoden nicht eindeutig zu beweisen war, führten wir den Schultz-Dale-Test nach aktiver Immunisierung mit Rh-positiven Erythrocyten und passiver Immunisierung sowie passiver Sensibilisierung in vitro mit gereinigtem Anti-D durch. Diese Methode, bei der die Anaphylaxie, ausgelöst durch eine Antigen-Antikörper-Reaktion, zur Kontraktion der Uterusmuskulatur des sensibilisierten Meerschweinchens mit nachfolgender Desensibilisierung führt, eignete sich sehr 
gut zur Prüfung dieser Fragestellung. Bei exakter Versuchsanordnung gibt diese Methode eine spezifische Information, da die Muskelkontraktion nur durch korrespondierende Antigene ausgelöst werden kann und durch Antihistaminika verhindert wird.

\section{Material und Methoden}

Die Isolierung des kleinmolekularen Proteins wurde aus Blutkonserven mit den Phänotypen CCDee, ccDEE, CcDEe, CcDee, $\mathrm{ccDEe}, \mathrm{ccDee}$ gesondert vorgenommen. Konserven des Phänotyps ccdee wurden als Kontrolle demselben Verfahren unterworfen. Die serologische Testung der Erythrocyten erfolgte in der Objektträgerstandardtechnik mit Antiseren von Molter, Heidelberg. Die 180 Blutspenderkonserven der Versuchsreihe wurden uns von der Blutbank (Leiter: Dr. KLUGE) zur Verfügung gestellt. Das Isolierungsverfahren, die durchgeführten Homogenitätsprüfungen des Proteins nach Anti-D-Immunadsorption und seine biochemische Charakterisierung wurden in der ersten Mitteilung ausführlich dargestellt (1).

\section{Hämagglutinations-Hemmtest}

Der Hämagglutinations-Hemmtest wurde nach der konventionellen Methodik durchgeführt. Das Anti-D-Serum war auf den Titer eingestellt, bei dem Rh-positive papainisierte Erythrocyten noch maximal agglutiniert wurden. Antigen-Testungen wurden durchgeführt mit Diffusat I/II nach Gefriertrocknung in 1,5 und 10proz. Lösung bei Verwendung von $0,1 \mathrm{ml} 2$ proz. Suspension papainisierter Erythrocyten in 0,9proz. $\mathrm{NaCl}$-Lösung und 0,1 ml Anti-DSerumverdünnung. In gleichen Konzentrationen wurden die Protein-haltigen Fraktionen I nach Sephadex-Gelfiltration und Refiltration und die Substanzen, die von dem Immunadsorbens, bestehend aus Anti-D, gekoppelt mit PAB-Cellulose, sowohl mit Wasser als auch mit $0,1 \mathrm{M}$ Glycinpuffer bei $\mathrm{pH} 2,5$ eluiert worden waren, getestet.

Der Hämagglutinations-Test nach Adsorption wurde mit $0,5 \mathrm{ml}$ nativem Stroma oder der gewichtsäquivalenten Menge des lyophilisierten Stromas vorgenommen. Hierbei wurden $0,5 \mathrm{ml}$ Anti-D mit dem bei dem Hämagglutinations-Hemmtest verwandten Titer $30 \mathrm{Min}$. bei $37^{\circ}$ mit Stroma inkubiert, danach abzentrifugiert und in dem Uberstand die Anti-D-Reduktion im Hämagglutinations-Test mit papainisierten Erythrocyten in einer Verdünnungsteihe bestimmt.

Die passive Hämagglutination wurde nach der Methode von GoLD und FUDENBERG (2) sowohl im Röhrchentest als auch auf Objektträgern durchgeführt, in der entweder das Protein aus $\mathrm{Rh}$-positiven Erythrocyten oder Anti-D-IgG auf rh-negative Erythrocyten durch Chrom-(III)-chlorid gekoppelt worden war. In Vergleichsuntersuchungen wurde der passive HämagglutinationsTest an tannierten Erythrocyten nach der Methode von BoYDEN (3) ausgeführt.

Isolierung an Immunadsorbentien wurde an PAB-Cellulose (4), die durch Diazotierung mit Anti-D gekoppelt worden war, vorgenommen, Vergleichsuntersuchungen wurden mit Aminostyrol (5) und DEAE-Cellulose, behandelt mit Glutaraldehyd (6), durchgeführt (vgl. l. c. (1)).

SCHUITZ-DALE-Test

Versuchsanordnug $(7-13,22)$ : Die apparative Anordnung ist aus Abbildung $1 \mathrm{zu}$ ersehen.

\section{Reagenzien}

1. Tyrode-Stammlösung (12, 13): $\mathrm{KCl}: 2,90 \mathrm{~g} / \mathrm{l} ; \mathrm{MgCl}_{2} \cdot 6 \mathrm{H}_{2} 0$ : $4,26 \mathrm{~g} / \mathrm{l} ; \mathrm{NaHCO}_{3}: 20,3 \mathrm{~g} / \mathrm{l} ; \mathrm{NaCl}: 160,0 \mathrm{~g} / 1 ; \mathrm{CaCl}_{2} \cdot 6 \mathrm{H}_{2} \mathrm{O}$ : $5,752 \mathrm{~g} / \mathrm{l}$.

2. Tyrode-Gebrauchslösung: Je $100 \mathrm{ml}$ Tyrode-Stammlösung werden auf 21 mit sterilem dest. Wasser aufgefüllt und mit $2,0 \mathrm{~g}$

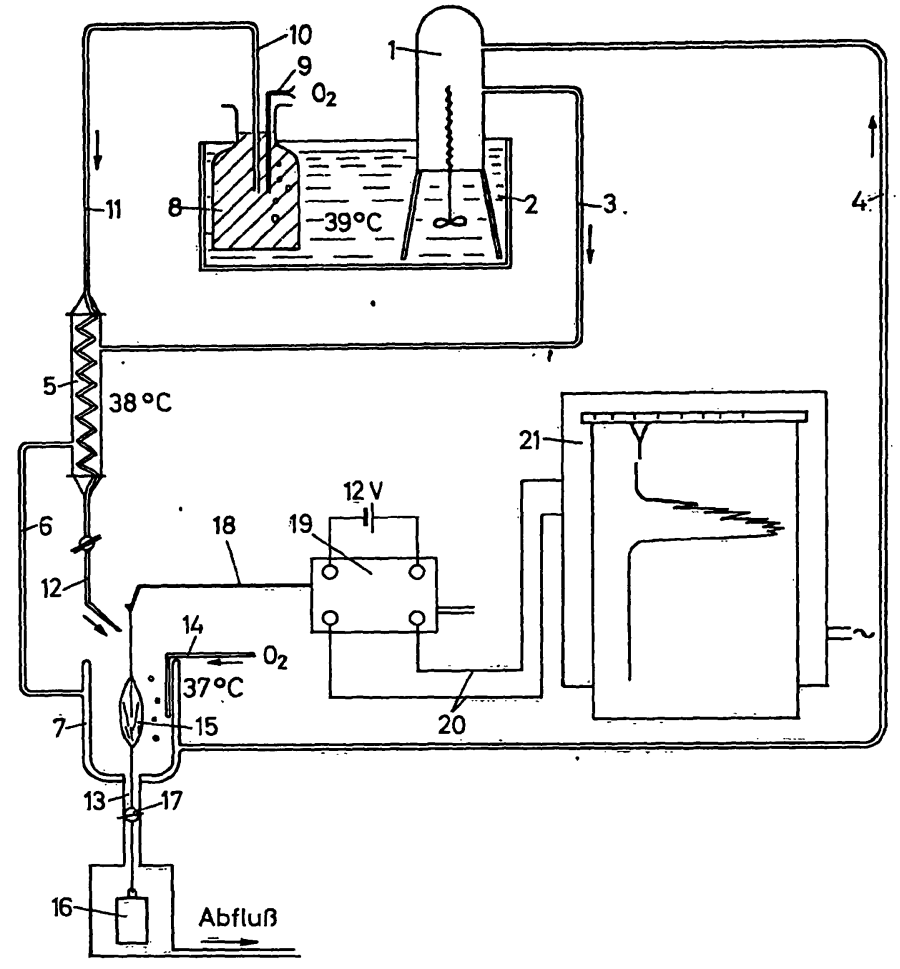

Abb. 1

Versuchsanordnung für die Testung der ScHulTz-DALE-Reaktion, modifiziert nach B. C. ADELMANN Max-Planck-Institut für Eiweißund Lederforschung, München
1 Wasserumlaufpumpe
2 Wasserbehälter
3 Ablauf Wasserpumpe
4 Einlauf Wasser
6 Liebig-Kühler
6 Verbindung
7 Muskelbad
8 Vorratsbeh
9 Einlaß $\mathrm{O}_{2}$ Tyrode-Lösung
11 Ablauf für Tyrode-Lösung
12 Einlauf für Tyrode-Lösung
3 Ablauf Muskelbad
14 Einlaß $\mathrm{O}_{2}$ für Muskelbad
5 Muskel
6 Muskelvorspanngewicht $500 \mathrm{mg}$
7 Arretierung für Muskelgewicht
8 HSE Biegestab N
9 Transducer
Verbindung Modulator/Schreiber 21 Schreiber

D-(+)-Glucose versetzt. Die Tyrode-Gebrauchslösung wird auf pH 7,4 eingestellt und mit $\mathrm{O}_{2}$ durchperilt.

3. Histamindihydrochlorid 0,1proz., Firma Gattiker, Zürich.

4. Ilvin (1-(p-Bromphenyl)-1-(2-pyridyl)-3-dimethylpropanmaleat), Fa. Merck, Darmstadt, als Stammlösung 0,1 proz. verdünnt mit 0,9proz. NaCl-Lösung.

5. Human-Albumin (Fa. Behring, Marburg) batch-Nr.: 2936.

6. Anti-Human-Albumin-Serum vom Kaninchen (Fa. Behring, Marburg) batch-Nr.: $1585 \mathrm{H}$ und $1585 \mathrm{~W}$.

7. Rinderalbumin, reinst $98 \%$, kristallin (Fa. Serva, Heidelberg). 8. Anti-D-IgG, IgG III nach Roexcke und JUNGFer (19), Titer $1 / 512$.

9. Antiseten (Dr. Molter GmbH, Heidelberg).

Das Muskelpräparat wurde aus dem oberen bis mittleren Meerschweinchenuterushornsegment entnommen und bei Raumtemperatur in einem mit $\mathrm{O}_{2}$ durchströmten Tyrode-Bad bis zum Versuchsbeginn gewaschen und aufbewahrt. Das Muskelsegment wurde am proximalen Ende an der Aufhängevorrichtung des Biegestabes ${ }^{3}$ ) befestigt, so daß es vollkommen in das Temperatur-

3) HSE-Biegestab N (Fa. Hugo Sachs, Hugstetten/Br.). 
$\left(37^{\circ} \pm 0,1\right)$ und $\mathrm{pH}$-konstante $(7,4 \pm 0,2)$ Tyrode-Bad, das kontinuierlich mit $\mathrm{O}_{2}$ durchströmt war, eintauchte. Die Muskelausgangsspannung wurde durch ein Gewicht von $500 \mathrm{mg}$ konstant gehalten, das an dem distalen Ende des Muskels befestigt war und eine isotonische Arbeitsweise ermöglichte. Die mechanischen Kontraktionsimpulse wutden über den Biegestab des Transducers in elektrische Impulse umgewandelt, die auf einem Schreiber ${ }^{4}$ ) bei einem Papiervorschub von $2 \mathrm{~cm} / \mathrm{Min}$. registriert wurden. Als Energiequelle des Transducers wurde ein 12-VAkkumulator verwandt. Nachdem die Null-Linie mindestens $10 \mathrm{Min}$. konstant war, wurde die Muskelkontraktion mit Histamindihydrochlorid standardisiert, bis die Ausschläge nach Applikation von $10-0,2 \mu \mathrm{g}$ Histamindihydrochlorid in abfallenden Dosen annähernd gleich groß waren. Zwischen jeder HistaminTestung wurde die Tyrode-Lösung des Muskelbades erneuert und die Muskulatur mit der Tyrode-Lösung gewaschen. Nach Standardisierung der Testmuskulatur wurden folgende Versuche durchgeführt:

1. Aktive Immunisierung mit $0 \mathrm{Rh}$-positiven und 0 rh-negativen Erythrocyten (14): Erythrocyten des Phänotyps CcDEe und ccdee, zweimal $1 \mathrm{ml} 50 \mathrm{proz}$. Suspension, wurden den Meerschweinchen i. p. in Abständen von 5 Tagen injiziert. In einem zweiten Versuchsansatz wurde mit dem Protein, gewonnen von Rh-positiven Erythrocyten, nach folgendem Schema aktiv immunisiert: Die Gesamtproteinmenge betrug $15 \mathrm{mg}$, von der bei der ersten Injektion $5 \mathrm{mg}$, danach 4, 3, 2 und $1 \mathrm{mg}$ im Abstand von je 3 Tagen als 0,5 proz. Lösung in 0,9 proz. NaCl-Lösung und komplettem Freundschem Adjuvans = 1:1 (v/v) i. m. appliziert wurden. Die Antikörper-Testung erfolgte nach Serumgewinnung am 21. Versuchstag, Komplementinaktivierung und nach Adsorption an 0 ccdee-Erythrocyten durch Titerbestimmung gegen $0 \mathrm{Rh}$-positive papainisierte Erythrocyten.

Die Antigen-Antikörper-Reaktion wurde im Schultz-Dale-Test folgendermaßen geprüft: Das Muskelsegment des aktiv durch Erythrocyten oder Membranprotein immunisierten Tieres wurde in das Temperatur- $\left(37^{\circ} \pm 0,1\right)$ und $\mathrm{pH}$-konstante $(7,4 \pm 0,2)$ Muskelbad von $5 \mathrm{ml}$ Inhalt gebracht; nach Einstellung der geforderten Null-Linie wurde mit Histamindihydrochlorid standardisiert, und danach wurde dem Muskelbad $1 \mathrm{mg}$ Protein von th-negativen bzw. Rh-positiven Erythrocyten zugesetzt. Nach erfolgter Muskelkontraktion wurde zur Prüfung der Desensibilisierung die gleiche Menge des verwandten Proteins appliziert. Anschließend wurde die Reaktionsfähigkeit des Muskelsegmentes mit Histamin überprüft. Nach Zugabe von $10-20 \mu \mathrm{g}$ Antihistamin und anschließendem Wechsel der Tyrode-Lösung wurde getestet, ob die Histamin-Reaktion und die durch die AntigenAntikörper-Reaktion hervorgerufene Anaphylaxie durch das Antihistaminikum aufgehoben werden konnte (15-18). Nach aktiver Immunisierung mit $15 \mathrm{mg}$ Albumin pro Meerschweinchen bei demselben Immunisierungsschema wurde unter gleichen Versuchsbedingungen mit $1 \mathrm{mg}$ Albumin als Antigen die anaphylaktische Reaktion, Desensibilisierung und der Anti-HistaminEffekt an diesem Antigen-Antikörper-System getestet.

2. Passive Immunisierung: Sie wurde mit Anti-D, das über DEAECellulose- und Hydroxylapatit-Säulen gereinigt worden war, durchgeführt (19). 0,5 ml dieses Anti-D-IgG wurden zweimal innerhalb von 2 Tagen vor Durchführung der Schurtz-DaleReaktion dem Tier i. p. injiziert. Präparation des Muskelsegmentes und Testung des Antigens erfolgte analog der aktiven Immunisierung mit je $1 \mathrm{mg}$ Protein von rh-negativen und $\mathrm{Rh}$-positiven Erythrocyten. Die Desensibilisierung und Antihistamin-Wirkung auf den Versuchsablauf wurde in gleicher Weise geprüft.

3. Passive Sensibilisierung in vitro: Sie erfolgte durch Inkubation der Muskulatur in $1 \mathrm{ml}$ Anti-D-IgG-Tyrode-Lösung $(1: 4, v / v)$ für $100 \mathrm{Min}$. bei $37^{\circ}$ unter $\mathrm{O}_{2}$-Zufuhr $(18,20,21,22)$. In der gleichen Versuchsanordnung wie in 1. und 2. wurden jeweils $1 \mathrm{mg}$ Protein aus rh-negativen und Rh-positiven Erythrocyten getestet. Neben dem geteinigten Membranprotein wurde die Schultz-DaleReaktion mit $0,2 \mathrm{ml}$ nativem Stroma bzw. der gewichtsäqui-

4) Schreiber Linecomp (Laufgeschwindigkeit $2 \mathrm{~cm} / \mathrm{Min}$.). valenten Menge lyophilisierten Stromas durchgefühtt. Sowohl bei der aktiven als auch bei der passiven Immunisierung und der Sensibilisierung in vitro durch Anti-D wurden Human-Albumin und Rinder-Albumin als nicht korrespondierende Antigene zur Überprüfung der antigenspezifischen Auslösung der Muskelkontraktion und Desensibilisierung verwandt. Bei der aktiven Immunisierung mit Albumin und der passiven Immunisierung sowie der Sensibilisierung in vitro mit Anti-Albumin wurden Rinder-Albumin, rh-negatives und Rh-positives Protein als nicht korrespondierende Antigene getestet.

\section{Ergebnisse}

\section{Agglutinations-Hemmtest}

Das Diffusat I/II, die proteinhaltige Fraktion I $\mathrm{Na}^{+}$, $\mathrm{K}^{+}$-Konzentration kleiner als $0,2 \%$ ), erhalten durch Sephadex-Gelfiltration und Refiltration, sowie das mit $0,1 \mathrm{M}$ Glycinpuffer bei pH 2,5 von dem Immunadsorbens eluierte Protein wurden mit papainisierten $\mathrm{Rh}$-positiven Erythrocyten bei einem Antiserum-Titer, der eine noch maximale Aktivität zeigte, im HämagglutinationsHemmtest untersucht. Hierbei fanden wir bei 1 proz. Konzentration des getesteten Materials trotz gleicher Präparationen und Versuchsanordnung eine sehr inkonstante Anti-D-Hemmung von 0 bis 3 Titerstufen. Die Zugabe von Lecithin, isoliert sowohl aus dem Stroma von Rh-positiven und rh-negativen Erythrocyten als auch ex ovo lösten eine unspezifische Steigerung des Hemmtiters um mehrere Stufen aus. Ein ähnlicher Effekt konnte auch bei unphysiologischer Erhöhung der $\mathrm{Na}^{+}$- und $\mathrm{K}^{+}$-Konzentration beobachtet werden. Die passive Hämagglutination nach Gold und Fudenberg (2) mit Kopplung des Membranproteins oder Anti-D-IgG durch Chrom-[III]-Chlorid an rh-negative Erythrocyten und in der Versuchsanordnung nach BoydeN (3) mit tannierten Erythrocyten zeigte sowohl mit Anti-D als auch mit dem Protein von Rh-positiven Erythrocyten keine Agglutination. Bei nativem Stroma, das wir nach Hämolyse der Erythrocyten durch $24 \mathrm{stdg}$. Stehen bei $+4^{\circ}$ in dest. Wasser bei $\mathrm{pH}$ 6,4 und durch Waschen des Stromas bei $+5^{\circ}$ mit dest. Wasser von $\mathrm{pH} 6,4$ erhalten hatten, entsprach die quantitative Anti-D-Adsorption den Titerstufen, die wir bei nativen Erythrocyten beobachtet hatten. Nach Lyophilisation des Stromas war bei gewichtsgleichem Ansatz keine meßbare Anti-D-Adsorption des Stromas festzustellen. Eine ähnliche, wenn auch nicht so ausgeprägte Reduzierung der Antigenität konnten wir bei Senkung des $\mathrm{pH}$ unter 3,5 während der Aufarbeitung feststellen. Wurde das Membranprotein von CcDEe-Erythrocyten im HämagglutinationsHemmtest mit verschiedenen Antiseren geprüft, dann fanden wir in dieser Versuchsanordnung bei Verwendung von Anti-D eine Hemmung von $0-3$ Stufen, bei Anti-C von 2 Stufen, bei Anti-E von einer Stufe und bei Anti-A von einer Stufe, bei Anti-e war keine Hemmung festzustellen (Tab. 1). Die Untersuchungen der Rh-Untergruppen stützen sich jedoch nur auf einen Versuch. Das Protein von rh-negativen Erythrocyten löste keine Hemmung im Hämagglutinations-Hemmtest aus. 
Weicker, Kropp u. Roelcke: Kleinmolekularcs Erythrocytenmembranprotein mit Rh-(D)-Eigenschaft

Tab. 1

Hämagglutinations-Hemmtest mit papainisierten Rh-positiven Erythrocyten. Anti-D eingestellt auf die Konzentration, die papainisierte Rh-positive Erythrocyten gerade noch maximal agglutiniert. Iproz. Lösung des Membranproteins von Rh-positiven Erythrocyten in
dest. Wasser (Konserve 154/1/2). Säule F I

\begin{tabular}{|c|c|c|c|c|c|c|}
\hline \multirow{2}{*}{$\begin{array}{l}\text { Verdünnung } \\
\text { der I proz. } \\
\text { Membran- } \\
\text { proteinlösung }\end{array}$} & \multicolumn{6}{|c|}{$\begin{array}{c}\text { Hämagglutinationshemmung von } O R_{2} R_{2} \\
\text { papainisierten Testerythrocyten }\end{array}$} \\
\hline & Anti-D & Anti-C & Anti-E & Anti-c & Anti-e & Anti-A \\
\hline $\begin{array}{c}2 / 1 \\
1 / 2 \\
1 \% \\
1 \% 8 \\
1 / 10 \\
2 / s_{2} \\
\mathrm{VaCl}-\mathrm{K} \text { ontrolle }\end{array}$ & $\begin{array}{l}(++t) \\
++t \\
++t \\
++t\end{array}$ & $\begin{array}{l}+t \\
+t \\
+t \\
+t \\
++\end{array}$ & $\begin{array}{l}++t \\
++t \\
++t \\
++t \\
++t \\
+t+\end{array}$ & $\begin{array}{l}\text { hämolyt. } \\
\begin{array}{l}+++ \\
++t \\
++t \\
++t \\
+++\end{array}\end{array}$ & $\begin{array}{l}+t+ \\
++t \\
++t \\
++t \\
++t \\
++t \\
+t+\end{array}$ & $\begin{array}{l}t+t \\
++t \\
++t \\
+t+ \\
+t+\end{array}$ \\
\hline
\end{tabular}

\section{SCHULtZ-DALE-Reaktion}

$\mathrm{Da}$ der Hämagglutinations-Hemmtest nur eine sehr inkonstante Anti-D-Hemmung erkennen ließ und durch die passive Hämagglutination der Antigen-Charakter des Membranproteins nicht bewiesen werden konnte, führten wir die SCHULTZ-DALE-Reaktion nach der oben beschriebenen Technik aus. Insgesamt wurden 75 Versuche an Meerschweinchenuterushornsegmenten durchgeführt. Die Ergebnisse dieser Untersuchungsreihe sowohl nach aktiver und passiver Immunisierung als auch nach Anti-D-Sensibilisierung des Meerschweinchenuterushornes und die Vergleichsuntersuchungen mit Albumin sind in Tabelle 2 dargestellt. Sowohl nach aktiver Immunisierung des Versuchstieres durch Rhpositive Erythrocyten und deren isoliertem Membranprotein als auch bei passiver Immunisierung und Sensibilisierung in vitro mit gereinigtem Anti-D konnte durch $1 \mathrm{mg}$ Rh-positives Protein eine klassische Schultz-DaLe-Reaktion mit Desensibilisierung bei erhaltener Histaminempfindlichkeit der Muskulatur ausgelöst werden (Abb. 2a, b, 3c). Die Ergebnisse entsprechen den Resultaten, die wir mit Albumin bzw. Anti-Albumin als anerkanntem Antigen-AntikörperSystem in gleicher Versuchsanordnung gefunden hatten (Abb. $3 \mathrm{~b}$ ). Hingegen wurde durch das Protein, isoliert von th-negativen Erythrocyten, Human-Albumin und Rinder-Albumin nach passiver Immunisierung keine Anaphylaxie hervorgerufen, da es sich um nichtkorrespondierende Antigene handelte. Nach aktiver Immunisierung mit rh-negativen Erythrocyten konnte durch das isolierte rh-negative Protein eine SchultzDALE-Reaktion mit Desensibilisierung ausgelöst werden
(Abb. 4). Bei Verwendung von nativem Stroma $\mathrm{Rh}$ positiver Erythrocyten als Antigen fanden wir bei passiver Sensibilisierung eine Muskelkontraktion, die bei gewichtsgleicher Menge lyophilisierten Stromas jedoch ausblieb. Im Gegensatz zu dem Membranprotein trat nach Applikation von nativem Stroma Rh-positiver Erythrocyten keine Desensibilisierung ein. Das rhnegative Stroma verursachte nach passiver Sensibilisierung mit Anti-D in vitto keine, Muskelkontraktion. Von den insgesamt 75 durchgeführten SchultzDALE-Reaktionen nach aktiver und passiver Immunisierung sowie Sensibilisierung in vitro mit gereinigtem Anti-D waren 72 Versuche positiv und ließen die geforderte Desensibilisierung bei erhaltener HistaminEmpfindlichkeit erkennen. Die Applikation von Antihistaminica vor Antigen-Zugabe unterband die sonst konstant beobachtete anaphylaktische Reaktion $(15,16$, 17) (Abb. 5). Die drei negativen Versuchsergebnisse waren durch pH-Verschiebungen im Muskelbad und durch $\mathrm{O}_{2}$-Mangel $\mathrm{zu}$ erklären. Für die passive Immunisierung und Sensibilisierung in vitro ist es wichtig, $\mathrm{da} B$ nur hochgereinigtes Anti-D-IgG verwandt wird, das vergleichsweise nur geringe Mengen nicht Anti-Dspezifischer Immunglobuline enthält. Die schwache Muskelkontraktion des von Anti-D-Immunadsorbens gewonnenen Materials war durch die lange Exposition des Proteins bei einem $\mathrm{pH}$ von 2,5 zu erwarten, da auch native Erythrocyten und Stroma in diesem $\mathrm{pH}-B$ ereich erheblich an Antigenität verlieren. Unspezifische Muskelkontraktionen konnten mit Sicherheit ausgeschlossen werden, da nicht-sensibilisierte Muskelsegmente nach Applikation der hier verwandten Antigene keine anaphylaktische Reaktion zeigten (Abb. 3a). Die typische Scrultz-DaLE-Reaktion bei allen Versuchskonstellationen bei passiv und aktiv immunisierten Tieren war nur durch Applikation korrespondierender Antigene auszulösen. Lecithin, isoliert sowohl ex ovo als auch aus der Erythrocytenmembran, verursachte keine Anaphylaxie. Die Zugabe von aktivem komplementhaltigen Meerschweinchenserum allein bewirkte keine Muskelkontraktion (Abb. 2a, 3a, b, c, 4). In Gegenwart des korrespondierenden Antigens beschleunigte es die anaphylaktische Reaktion, ohne die Desensibilisierung zu stören oder den AntihistaminEffekt aufzuheben $(23,24,25)$ (Abb. 5).

Tab. 2

Zusammenstellung der durchgeführten ScHULTZ-DALE-Reaktionen

\begin{tabular}{|c|c|c|c|c|c|}
\hline Sensibilisierung & Material & $\begin{array}{l}\text { ScHULTZ-DALE-Versuch } \\
\text { keine Muskelkontrakt. } \\
\text { mit Desensibilisierung }\end{array}$ & $\begin{array}{l}\text { Muskelkontrakt. mit } \\
\text { Desensibilisierung }\end{array}$ & $\underset{\text { effekt }}{\text { Antihistamin- }}$ & Anzahl \\
\hline $\begin{array}{l}\text { Aktiv } \\
\text { Aktiv } \\
\text { Aktiv } \\
\text { Passiv } \\
\text { Passiv } \\
\text { Passiv in vitro } \\
\text { Passiv in vitro } \\
\text { Normaltier }\end{array}$ & $\begin{array}{l}\text { Rh-positive Erythrocyten } \\
\text { rh-negative Erythrocyten } \\
\text { Albumin } \\
\text { Anti-D } \\
\text { Anti-Albumin } \\
\text { Anti-D } \\
\text { Anti-Albumin } \\
\varnothing\end{array}$ & $\begin{array}{l}\text { rh-negatives Protein } \\
\text { Rh-positives Protein } \\
\text { Rinderalbumin } \\
\text { rh-negatives Protein } \\
\text { Rinderalbumin } \\
\text { rh-negatives Protein } \\
\text { Rinderalbumin } \\
\text { Rinderalbumin } \\
\text { Human-Albumin } \\
\text { rh-neg. u. Rh-pos. } \\
\text { Protein }\end{array}$ & $\begin{array}{l}\text { Rh-positives Protein } \\
\text { rh-negatives Protein } \\
\text { Human-Albumin } \\
\text { Rh-positives Protein } \\
\text { Human-Albumin } \\
\text { Rh-positives Protein } \\
\text { Human-Albumin } \\
\varnothing\end{array}$ & $\begin{array}{l}+ \\
+ \\
+ \\
+ \\
+ \\
+ \\
+\end{array}$ & $\begin{array}{l}15 \\
9 \\
6 \\
15^{* *} \\
11^{*} \\
7 \\
6 \\
6\end{array}$ \\
\hline
\end{tabular}

\footnotetext{
* abzüglich 1 Fehlkurve $\quad$ ** abzüglich 2 Fehlkurven. Erläuterungen s. Text.
} 

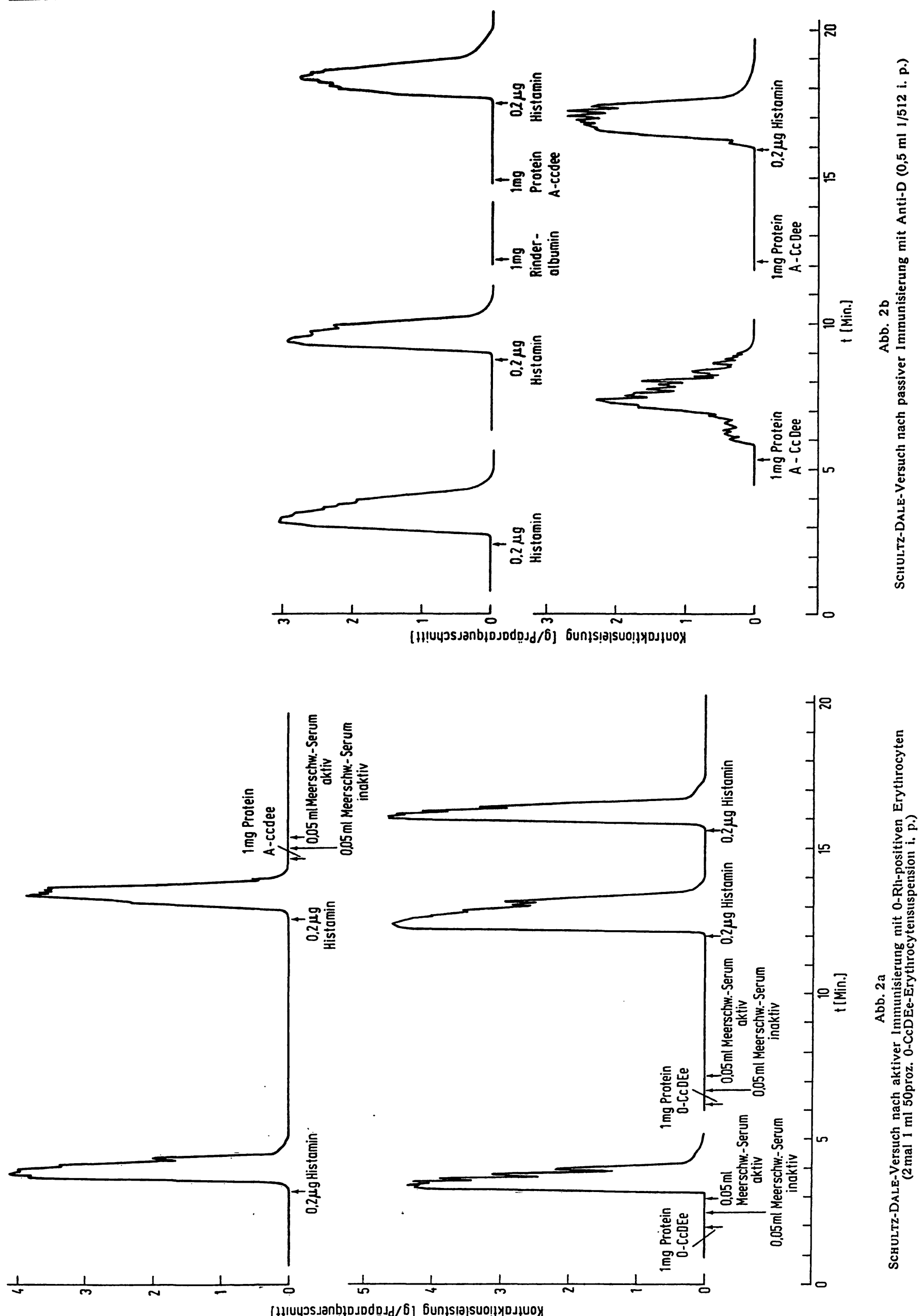

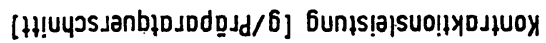



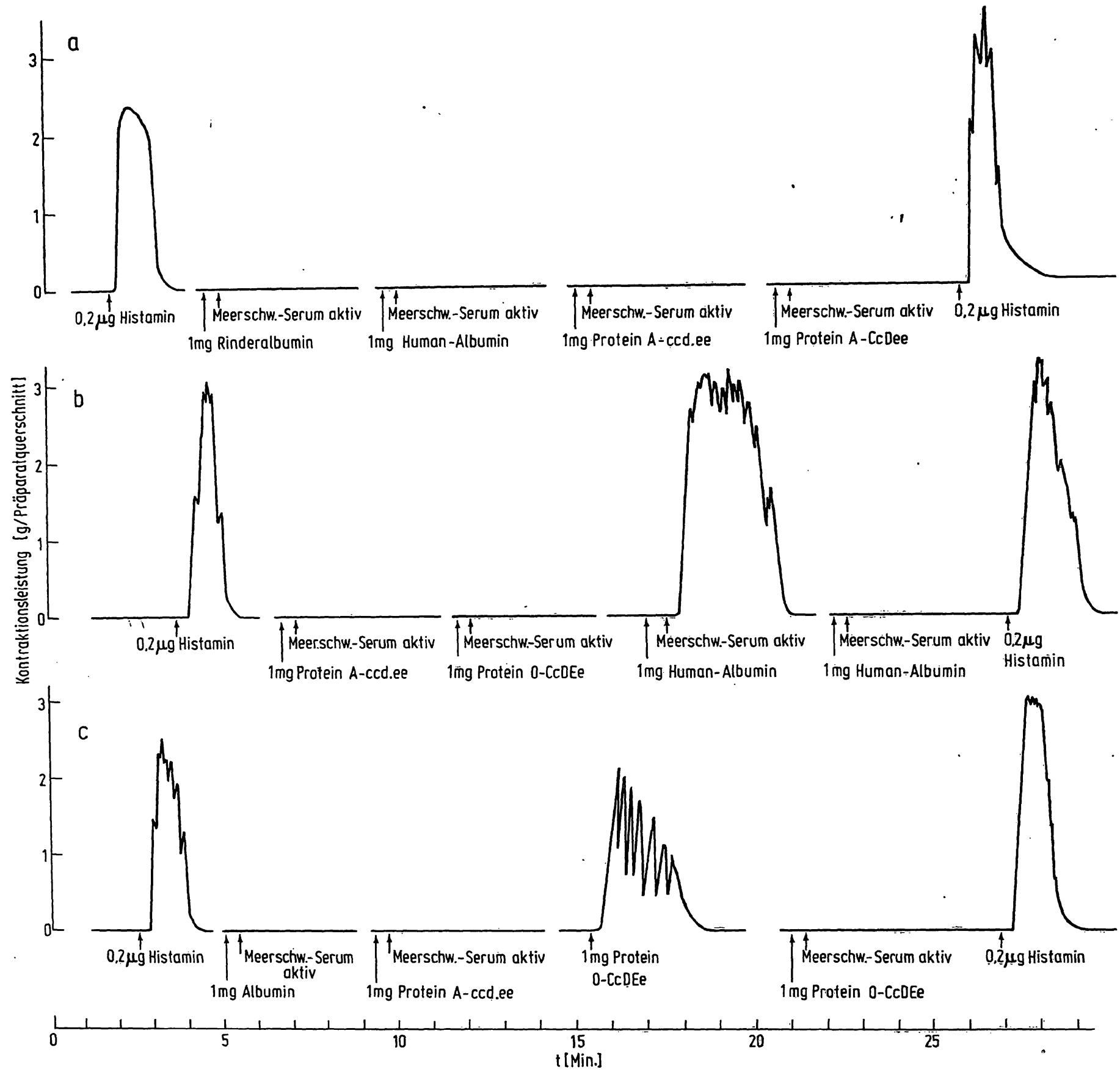

Abb. 3

SCHuLTZ-DALE-Versuch

a) Normaluterus nicht sensibilisiert.

b) Uterus sensibilisiert mit Anti-Albumin (in vitro, Anti-Albumin, $100 \mathrm{Min} ., 3^{\circ}$ )

c) Uterus sensibilisiert mit Anti-D (in vitro, Anti-D 1/512, 100 Min., 37 ${ }^{\circ}$ )

\section{Diskussion}

$\mathrm{Da}$ wir den Antigen-Charakter des kleinmolekularen Erythrocytenmembranproteins durch konventionelle serologische Methoden trotz seiner spezifischen Isolierung durch ein Anti-D-Immunadsorptionsverfahren nicht sicher beweisen konnten, benutzten wir die ScHultzDALE-Reaktion zur Klärung dieser Fragestellung. Die Kontraktion der Meerschweinchenuterusmuskulatur als Ausdruck einer anaphylaktischen Reaktion, ausgelöst durch eine Antigen-Antikörper-Reaktion mit Desensibilisierung des getesteten Muskelsegmentes, ist praktisch und theoretisch als Antigen-Nachweis an- erkannt $(7-13,22)$. Der Vorteil dieser Methode liegt in der Empfindlichkeit, die bei exakter Versuchsanordnung beweisend ist. Aussagen über quantitative Antigenoder Antikörperverhältnisse gibt sie nicht. Die Aufhebung der anaphylaktischen Reaktion durch Antihistaminica vor Applikation des korrespondierenden Antigens sowohl bei aktiv als auch passiv immunisierten Versuchstieren weisen darauf hin, daß. H-Substanzen für die Muskelkontraktion verantwortlich sein können, um so mehr, da auch das zur Standardisierung verwandte Histamindihydrochlorid nach Applikation von Antihistaminika zu keiner Mưskelkontraktion mehr 


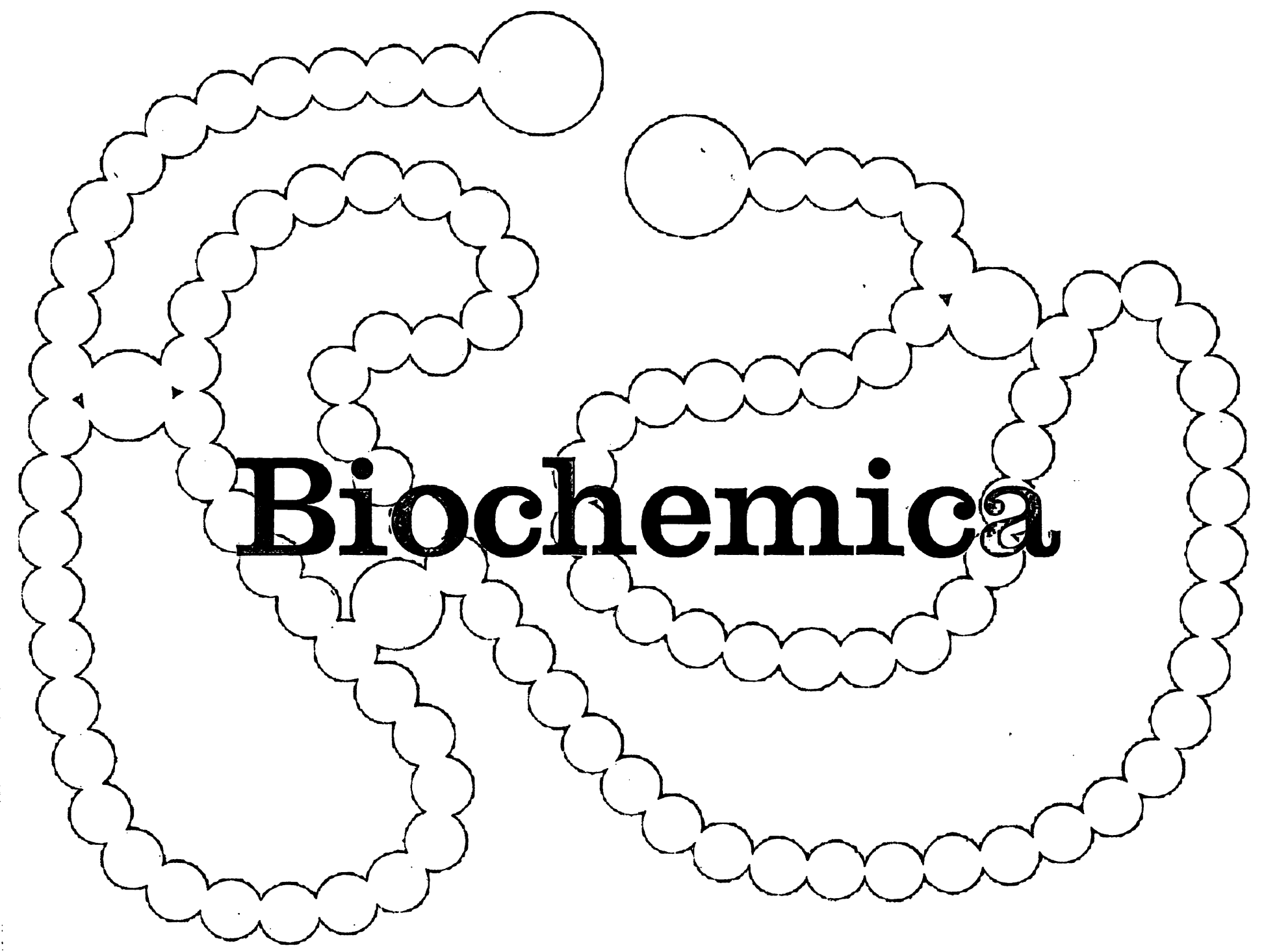

Polymer gebundene Enzyme

Bromelin - Chymotrypsin - Ficin - Papain - Pronase - Proteinase K (Tritirachium album) - Ribonuclease - Subtilisin - Trypsin (Rind) • Trypsin (Schwein) - Trypsin-Inhibitor (Sojabohnen)

Träger

Carboxymethylcellulose - Vernetztes Maleinsäureanhydrid

\section{Besondere Vorteile}

Hohe spezifische Aktivitäten - Enzymaktivität ausschließlich kovalent gebunden - Gleichbleibende Qualität durch Mindestaktivitätsangaben garantiert

Ausführliche Spezifikationen stehen auf Wunsch zur Verfügung. 


\section{Dünnschicht-}

\section{Chromatogramme}

gezielt entwickelln

\author{
mit der CAMAG \\ VARIO-KS-Kammer
}

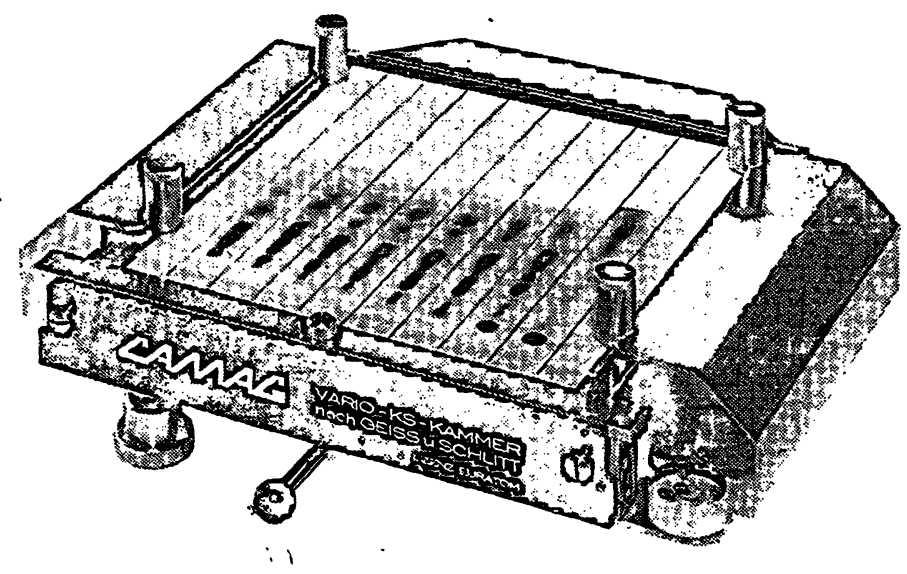

Reproduzierbare Chromatogramme besonders bei der quantitativen DC!

Entwicklung mit Schichtvorbeladung: optimale Trennergebnisse, vorausbestimmbares Trennverhalten.

Beeinflussung der Trenneigenschaften durch Längs- und Quergradienten.

Substanzklassenanalyse.

Ausbildung von Feuchtegradienten zur Überbrückung grosser Polaritätsunterschiede im Trenngemisch.

Durchlaufchromatographie.

Ausführliche Beschreibung in unserem Prospekt 251-400.

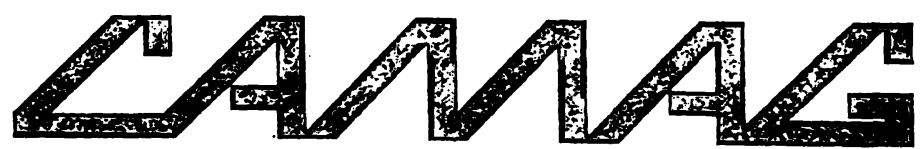

Homburgerstrasse 24 4132 Muttenz/Schweiz

Unser Zweigbetrieb in der Bundesrepublik:

1000 Berlin 45, Baseler Strasse 65

Führend in Dünnschicht-Chromatographie Dünnschicht-Elekrophorese TL 12 Hochspannungs-Elektrophorese

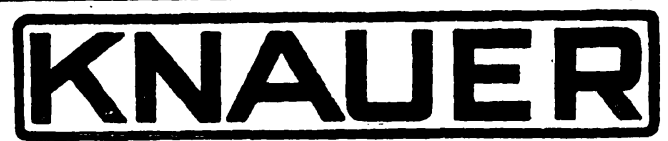

ELEKTRON IS CHES HALBMIKRO-OSMOMETER

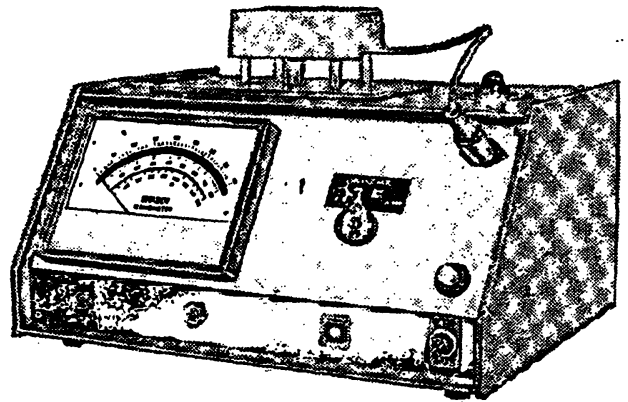

zur direkten Bestimmung der Osmolalität aller Körperflüssigkeiten wie Blut, Serum, Urin, Liquor durch Gefrierpunktmessung.

Kontrolle der Nierenfunktion

Kontrolle von isö-, hyper- und hypotonischen Lösungen Prüfung von Infusionslösungen

- Probevolumen nur $0,15 \mathrm{ml}$ oder $0,05 \mathrm{ml}$

- Betriebsbereitschaft sofort nach dem Einschalten

- Daver einer Messung ca. 2 Minuten

- Meßgenauigkeit 1 -2 Milliosmol/kg bzw: $1 \%$

- Preis DM 3600, - + MWSt

- Lieferung ab Lager oder laufenden Serien

Weitere Spezialität: Komplettes System zur Molekulargewichtsbestimmung zwischen 100 und 1000000 durch Kryoskopie, Dampfdruck-Osmomẹtrie und Membran-Osmometrie.

Wissenschaftlicher Gerätebau

KG. Dr.-Ing. Herbert Knaver \& Co. GmbH,

1 Berlin 37 (West), Holstweg 18, Tel. (0311) 848705

\section{BILTZ-KLEMM - FISCHER}

\section{Experimentelle Einführung in die anorganische Chemie}

Neu herausgegeben von W. KLEMM und W. FISCHER 63.-70. Auflage

Oktav. XII, 228 Seiten. 1970. Balacron flexibel DM 21,-

Die Kürzung der Dauer des Chemiestudiums führt besonders zu einer Kürzung der experimentellen Ausbildung, andererseits müssen im Ânfang grundlegende Stoffkenntnisse anhand der wichtigsten Ordnungsprinzipien vermittelt werden.

Um den heutigen Forderungen einer solchen Einführung Rechnung zu tragen, wurde das Werk überarbeitet und teilweise geändert. Während vom experimentellen Teil nur einige auf die analytische Chemie bezogene Versuche gestrichen wurden, erfuhren die theoretischen Abschnitte bei der Anpassung auf den heutigen Stand stärkere Änderungen. Neu gestaltet wurden insgesamt die Kapitel: Anwendungen des Massenwirkungsgesetzes, Oxidations-Reduktions-Potentiale. Bei der Behandlung von Säure-Basen-Gleichgewichten wurde der historischen Entwicklung von Davy und Liebig über Arrhenius und Ostwald zu Brönsted Rechnung getragen; im weiteren Verlauf werden aber nur noch die Brönstedschen Definitionen verwandt.

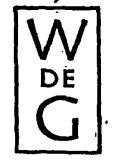


Abb. 4

SCHULTZ-DALE-Versuch nach aktiver Immunisierung mit 0-rh-negativen Erythrocyten (2 mal $1 \mathrm{ml} 50$ proz. 0-ccddeeErythrocyten-Suspension i. p.)
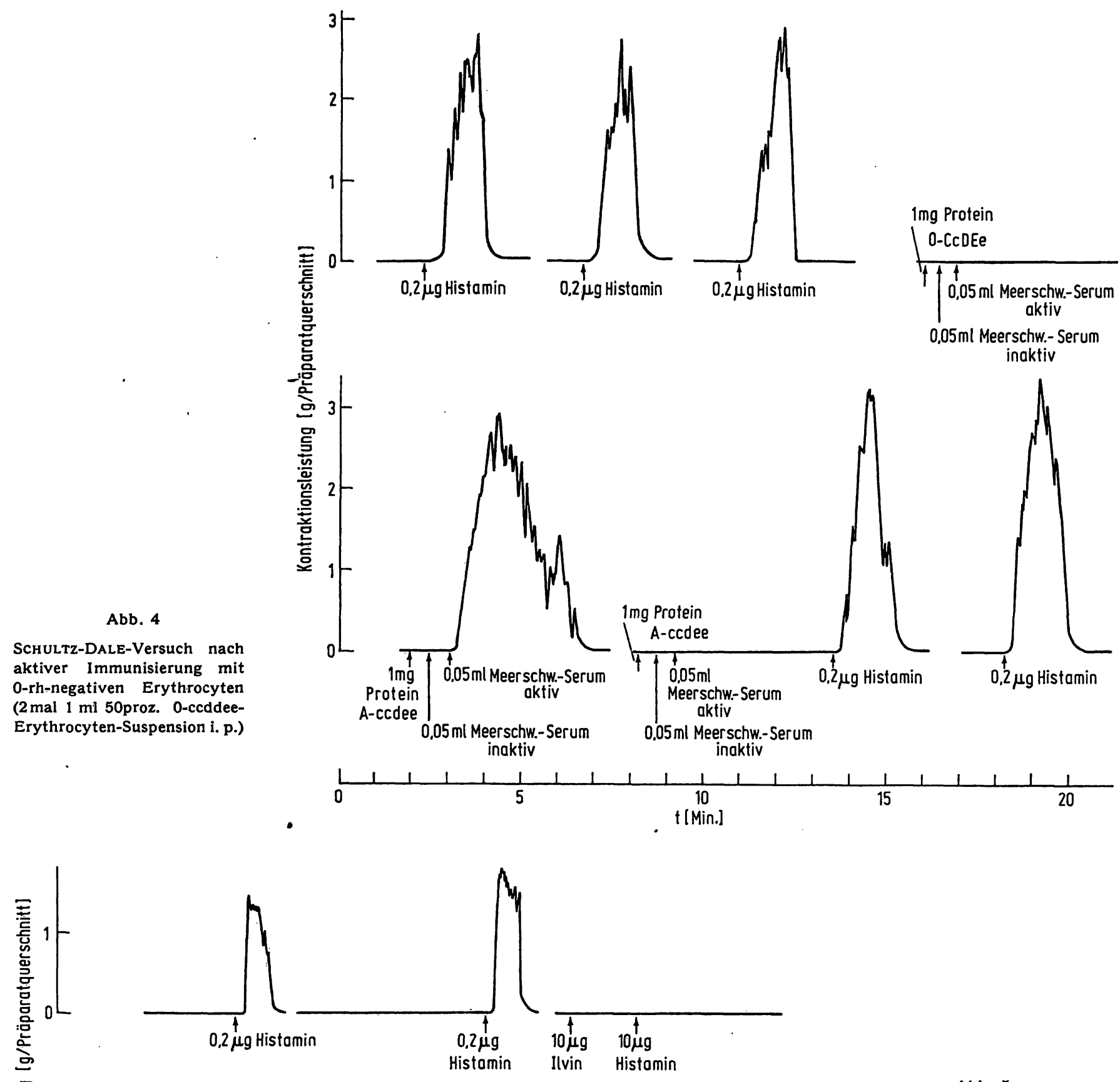

Abb. 5

Hemmung der Schultz-DAlE-Reaktion durch Antithistamin (aktive Immunisierung mit 2 mal $1 \mathrm{ml}$ 50proz. 0-CcDEe-ErythrocytenSuspension i. p.)

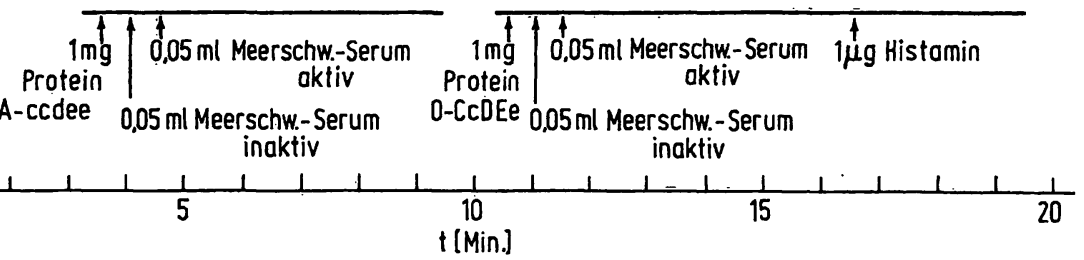

führte $(15,16,17)$. Diese Forderungen waren in allen Fällen sowohl nach aktiver und passiver Immunisierung als auch nach Sensibilisierung in vitro durch das von $\mathrm{Rh}$-positiven Erythrocyten isolierte Protein erfüllt (Tab. 2, Abb. 2a, b, 3c). Der Antigen-Charaktẹ dieses Proteins konnte weiterhin dadurch gestützt werden, $\mathrm{da}$ ß durch das Protein von rh-negativen Erythrocyten, Human-Albumin und Rinderalbumin nach passiver Immunisierung und Sensibilisierung mit gereinigtem Anti-D in vitro keine Schultz-DaleReaktion auftrat (Abb. 2b, 3c). Da die Ergebnisse bei der oben beschriebenen Versuchskonstellation identisch waren mit dem Albumin-Antialbumin-System, angewandt bei passiver und aktiver Immunisierung, kann in Analogie zu diesem anerkannten Immunsystem auch für das Membranprotein von Rh-positiven Erythrocyten und das Anti-D eine echte Antigen-Antikörper-Reaktion angenommen werden. Die strenge 
Antigen-Spezifität ist daraus zu ersehen, daß bei Versuchstieren, immunisiert mit rh-negativen Erythrocyten, keine Anaphylaxie durch Rh-positive Substanzen auftrat (Abb. 4). Gleiches gilt auch für die Versuchsanordnung mit aktiver Albumin- oder passiver Anti-Albumin-Sensibilisierung von Uterussegmenten (Abb. 3b). Als Hinweis für die D-Antigenität des Proteins kann auch angeführt werden, daß seine quantitative Ausbeute mit der D-Rezeptorenanzahl der einzelnen Phänotypen sehr gut korrespondierte $(26,27)$ und die letzte Stufe der Isolierung über eine Anti-DImmunadsorption erfolgte (vgl. 1. c. (1)). In Analogie zu dem Aktivitätsverlust von nativen Erythrocyten und nativem Stroma durch Gefriertrocknung oder Senkung des $\mathrm{pH}$ unter 3 ist es verständlich, daß wir durch die konventionellen serologischen Methoden nicht in der Lage waren, die Antigenität des Membranproteins schlüssig zu beweisen, da dieses Protein in der vielstufigen Aufarbeitung nur durch mehrfaches Lyophilisieren und $\mathrm{pH}-\mathrm{Senkung}$ isoliert werden konnte. Wahischeinlich ist das native zellständige Antigen dem isolierten, durch physiko-chemische Maßnahmen beeinflußten Antigen in der Konkurrenz um die combining-site des Antikörpers unterlegen, obwohl die
Protein-Inkubation vor Zugabe der Testerythrocyten als ausreichend angesehen werden muß. Da bei dem Schultz-Dale-Test die Antigen-Antikörper-Reaktion nicht in Gegenwart von nativen Rh-Rezeptoren der Erythrocytenmembran abläuft, ist auch theoretisch verständlich, $\mathrm{da} B$ wir mit $\operatorname{der}$ oben beschriebenen Versuchsanordnung eine Aussage über die Antigenität des Proteins erhielten. Im Hinblick auf die Molekülgröße von 6000-10000 mußten wir auf diese Methode zurückgreifen, da in der OuCHTERLONY-Technik oder einer ihrer Variationen bei den durchgeführten Versuchen keine Präzipitation beobachtet werden konnte, die auch nach theoretischen Überlegungen nicht sicher zu erwarten war. Die Ergebnisse der chemischen Chatakterisierung und der Untersuchungen über die Antigen-Eigenschaften der aus der Erythrocytenmembran isölierten Substanz mit der ScHultz-DaLeTechnik machen es wahrscheinlich, daß der Rh-Faktor (D) ein kleinmolekulares Protein ist. $\mathrm{Da}$ bereits zahlreiche Substanzen von verschiedenen Autoren als $\mathrm{Rh}$-Antigen beschrieben worden waren, sollten jedoch bis zur endgültigen chemischen Definition des Rh-(D) Antigens noch weitere Antigen-Nachweisverfahten durchgeführt werden.

\section{Literatur}

1. Weicker, H. und J. MetZ, diese Z., 9, 367 (1971). - 2. Gold, E. R. und H. H. FudenberG, J. Immunol., Baltimore 99, 859 (1967). - 3. BoYden, S. V., J. Exper. Med. 93, 107 (1951). 4. Brown, G. L. und A. K. Brown, Sym. Soc. Exp. Biol. XII, 6, Univ.-Press Cambridge (1958). - 5. Grubhofer, N. und L. SChLEIth, Hoppe-Seyler's Z. physiol. Chem. 297, 108 (1954). 6. Avranieas, St., B. Taudou und S. Chumlon, Immunochemistry 6, 67 (1969). - 7. Schultz, W. H., J. Pharmacol. Exper. Therap., Baltimore 1, 549 (1910). - 8. Schultz, W. H., J. Pharmacol. Exper. Therap., Baltimore 2, 221 (1910). - 9. DALE, H. H., J. Pharmacol. Exper. Therap., Baltimore 4, 167 (1912/13). 10. Coulson, E. J., J. Allergy, S. Louis 24, 458 (1953). - 11 NoAH, J. W., in: Immunological Methods ed. by J. F. Ackroyd. Blackwell Scientific Publications Oxford (1964). - 12. AdelmanN, B. C., persönliche Mitteilungen. 13. CampbEle, D. H., J. S. Garvey, N. E. Cremer und D. H. Sussdorf, Methods in Immunology, W. A. Benjamin, Inc. New York-Amsterdam (1963). 14. MÁjSKর́, A., M. KREčKoví, M. MaCha čKoví und R. Ziková, Zschr. Immunit. Allerg.forsch., 127, 129 (1964). - 15. WoLsteN-
HOME, G. E. W. und C. M. O'ConNor, Histamine, Ciba Foundation Symposion Churchill Ltd. London (1956). - 16. Cochrane, C. G. und H. J. Müller-Eberhard, J. Exper. Med. 127, 371 (1968). - 17. Mongar, J. L. und H. O. Schmed, J. Physiol. 135, 301 (1957). - 18. Austen, K. R und E. L. Becker, Biochemistry of the acute allergic teactions, Blackwell Scientific Publikations, Oxford and Edinburgh (1968). - 19. RoELCKE, D. und $H$. JungFer, Klin. Wschr. 48, 752 (1970). - 20. HALPERN, B. N., P. Liacopoulos, M. Liacopoulos-Briot, R. Binaghi und F. vaN NeER, Lmmunity Bull., 2, 351 (1959). - 21. Nrelsen, C. B., G. Terres und G. A. Feigen, Science 130, 41 (1959). 22. KABAT, E. A. und M. M. MAYER, Experimental Immunochemistry, Charles C. Thomas, Springfield, Ill. (1961). - 23. Jensen, J., Science 155, 1122 (1967). - 24. OsLer, A. G., H. G. Randall, B. M. Hirl und Z. Ovary, J. Exper. Med. 110, 311 (1959). - 25. Barbaro, J. F., J. Immunol., Baltimote 86, 377 (1961). - 26. Rosenfieid, R. E. und S. Kochwa, J. Immunol., Baltimore 92, 693 (1964). - 27. Rochina, E. und N. C. HughesJONES, Vox Sang. 10, 657 (1965).
Prof. Dr. H. Weicker 6900 Heidelberg Hospitalstr. 3 